\title{
Diseño e Implementación de un Prototipo de Electroeyaculador Escalable para uso en más de una especie
}

\section{Design and Implementation of a Prototype Electroejaculator Scalable for use in more than one Species}

\author{
Julián Molina Mosquera 1, Carolina Polo Caquimbo² y Mayra Alejandra Tovar \\ Clavijo $^{3}$
}

Resumen

El presente artículo describe la realización de un prototipo electrónico que facilita la aplicación de la técnica de electroeyaculación, la cual emplea estímulos eléctricos para producir una erección y posterior eyaculación del animal sobre el cual se efectúa el procedimiento, propiciando la preservación de material genético de alta calidad. Éste prototipo está constituido por una fuente generadora de impulsos eléctricos, con valores de frecuencia y voltaje específicos para cada especie. Su funcionamiento se basa en la aplicación de un estímulo a través de un transductor que consiste en un dispositivo de forma anular de proporciones requeridas según la especie y que mediante electrodos permite el paso dela energía eléctrica para provocar estímulos, ocasionando la eyaculación delanimal.

Palabras clave: electroeyaculación; estímulos eléctricos; transductor; genética

\begin{abstract}
The present article of investigation describes fully the accomplishment of an electronic prototype that facilitates the application of the electroeyaculación technique, which uses electrical stimuli to produce an erection and later ejaculation, of the animal on which the procedure is effected, propitiating the preservation of genetic material of high quality. This prototype is constituted by a generating source of electrical impulses, with specific values of frequency and voltage for every species. Its functioning is based on the application of a stimulus across a transducer that consists of a device of annul form of proportions needed according to the species and that by means of electrodes allows the step of the electric power to provoke stimuli, causing the ejaculation of the animal.
\end{abstract}

Keywords: electroejaculation; electrical impulses; transducer; genetics

\footnotetext{
1 Ingeniero Electrónico, Docente Universidad Surcolombiana - Neiva. Av. Pastrana-Carrera 1.Julián.

molina@usco.edu.co 2Ingeniero Electrónico. Universidad Surcolombiana-Neiva.Av.Pastrana-Carreral. carolinapolo@hotmail.com

3IngenieroElectrónico.UniversidadSurcolombiana-Neiva.Av.Pastrana-Carrera1.maytoO11787@hotmailco
} 


\section{Introducción}

La conservación de la genética se ha podido lograr gracias a los avances de biotecnologías reproductivas, entre éstas se destaca la criopreservación de las células espermáticas a bajas temperaturas, lo cual ha permitido diseminar las características deseables e individuales de machos genéticamente superiores en los sistemas de producción para diferentes especies, sistemas que han comenzado a cumplir con los estándares requeridos por los mercados internacionales. (Barrios, 2009). La electroeyaculación es una técnica que permite incrementar la productividad de las especies, asegurar la conservación de los recursos genéticos, difundir material genético de alto valor comercial, además de reducir riesgos en la transmisión de enfermedades. Dicha técnica se basa en la respuesta de erección -eyaculación como producto de estímulos eléctricos aplicados a través de un transductor por vía rectal, que activa el eje del sistema nervioso relacionado con el tracto reproductivo (Escobar, 2010).

La técnica de electroeyaculación fue utilizada primeramente en Australia. Está basada en la aplicación rítmica de un estímulo eléctrico por vía transrrectal estimulando el sistema nervioso autónomo y somático, que conduce a la obtención de secreciones delas glándulas accesorias y finalmente a la eyaculación.

El uso de tecnologías de última generación como herramientas aplicadas a las técnicas de reproducción asistida, permiten obtener una mayor precisión y fidelidad en los resultados, lo cual genera en una especie dada, un incremento de la velocidad de progreso genético mediante un acortamiento del intervalo generacional, obteniéndose de esta forma especímenes de alta calidad (Díaz atal, 2003).

El propósito de este proyecto fue diseñar e implementar un electroeyaculador escalable para uso en más de una especie que compite en funcionalidad y costo con los dispositivos existentes en el mercado, con el objetivo de brindar una opción a los pequeños productores pecuarios al tener un mayor aprovechamiento de sus animales utilizando técnicas como la inseminación artificial y la conservación de la genética de sus mejores ejemplares, ventajas que les brinda el método de la electroeyaculación.

\section{Metodología}

El prototipo tiene establecido dentro de sus modos de funcionamiento los valores eléctricos y de tiempo adecuados para usarse en las especies bovina, caprina y ovina de modo seguro, datos estipulados en la tabla 1.

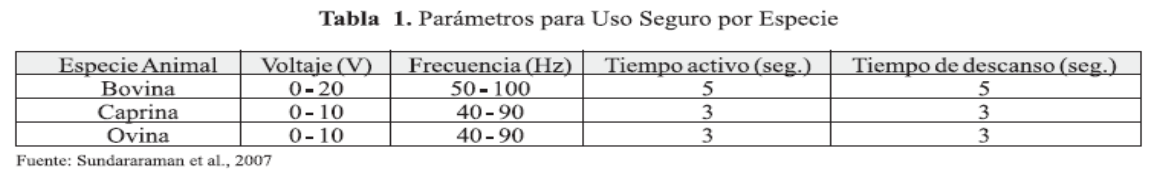

Las especies se eligieron, al considerar que la región surcolombiana es una zona de gran producción pecuaria, destacada por su alta calidad, lo que justifica el uso del Electroeyaculador para contribuir con estudios genéticos que permitan la preservación delas características más sobresalientes delas diferentes especies.

\subsection{Descripción General del Dispositivo}

De forma funcional las características con las que cuenta el Electroeyaculador, de aquí en adelante, llamado 'RAYTECH Multispecies Electroejaculator' son: Visualización del nivel del voltaje aplicado, frecuencia de trabajo, tiempos de descanso y actividad y medidor de batería; esto, a través de una pantalla de cristal líquido (LCD), indicadores luminosos de Power y Pausa, 6pulsadores para establecer la forma de trabajo del dispositivo, cargador de batería integrado y una sonda para trabajar sobre ovinos y caprino 
Para el desarrollo del Electroeyaculador RAYTECH fue necesario dividir el trabajo en cuatro bloques funcionales, los cuales son presentados en la Figura 1. Las características principales de cada módulo son:

- Fuentes de Alimentación: este módulo es el encargado de suministrar la energía necesaria a cada dispositivo que hace parte de El Electroeyaculador, donde una batería de 12 voltios actúa como alimentación principal y a través de otros dispositivos entrega voltajes de $5 \mathrm{~V}, 12 \mathrm{~V}$ y $-12 \mathrm{~V}$ a diferentes potencias según la etapa a alimentar.

- Unidad Central de Procesamiento: este módulo es elque dirige todo el prototipo, está encargado de recibir la información proveniente de los pulsadores mediante los cuales se configura el dispositivo para que funcione de una manera específica. Aquí se procesa la información suministrada y de acuerdo a ella genera la señal, además, muestra de manera visual dicha configuración a través de la pantalla LCD.

- Acondicionador de Señal: este módulo realiza una preamplificación de la señal generada en la unidad central de procesamiento, que permite a dicha señal variar en amplitud dentro de un rango de $\mathrm{O}$ a 10 voltios.

- Etapa de Potencia: este módulo realiza una amplificación tanto en voltaje como en corriente en un rango de Oa40voltios, con una corriente de salida de hasta 1amperio. El proceso se realiza porque esta etapa es la que se conecta directamente a los electrodos de la sonda rectal para la estimulación del animal.

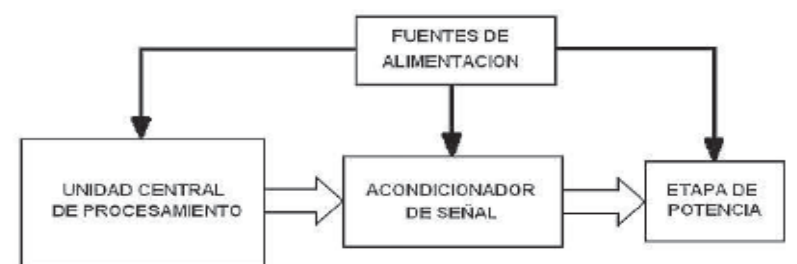

Figura 1. Bloques Funcionales de El Electroeyaculador RAYTECH

\subsection{Metodología de Fabricación}

Finalizada la etapa del diseño, se procedió con la fabricación física del prototipo, para ello, se hizo uso del programa PROTEUS de la firma LABCENTER ELECTRONICS en su versión 7.7; elcualademás de permitir realizar las simulaciones y pruebas de cada parte de la tarjeta, cuenta con la herramienta para elaborar el circuito impreso. Elaborado el diagrama esquemático del circuito impreso de la tarjeta de adquisición en este programa; el siguiente paso fue quemarla en una baquela de fibra de vidrio, abrir orificios y por último realizar la soldadura de los elementos, de esta manera se fabricó la tarjeta que da solución a los requerimientos de diseño. En la Figura 2 se presenta el resultado de esta fase de diseño.

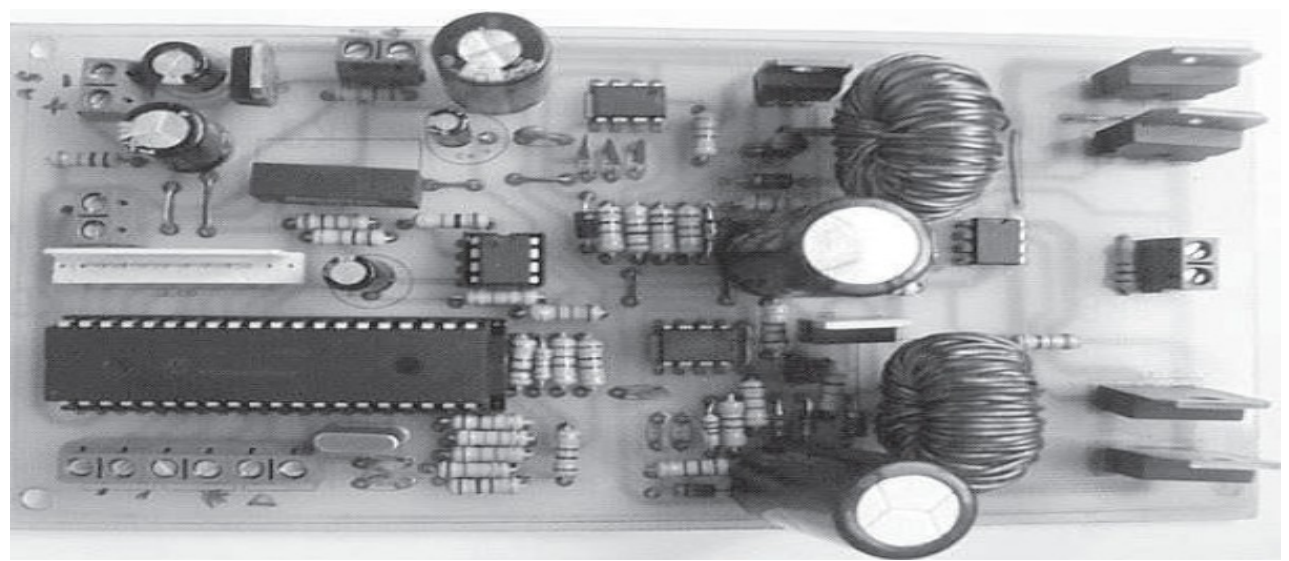

Figura .2. Montaje Circuito Placa Principal 
Además de la tarjeta principal de El Electroeyaculador, fue necesario realizar dos tarjetas adicionales para completar et dispositivo. En la primera se encuentran alojados los pulsadores e interruptores con los que se controla Et Electroeyaculador y los elementos que permiten la visualización del estado y las acciones realizadas.

La segunda tarjeta es la que permite realizarla carga de la batería principal junto con un transformador. El transformador se conecta directamente a la red para reducir el voltaje de $120 \mathrm{~V}$ a $12 \mathrm{VAC}$, de allí se conecta a los bornes de entrada de la tarjeta de carga, la cual se encarga de rectificar y filtrar ésta tensión y obtener el nivel de voltaje DC necesario para realizar el proceso de carga de la batería.

\subsection{Diseño y Construcción del Transductor para Eyaculación en Ovinos y Caprinos}

ElElectroeyaculador trabaja en conjunto con los electrodosque son los actuadores finales con los cuales se realiza la estimulación a los animales. Para el diseño y construcción del transductor, que fundamentalmente es el elemento que sirve de enlace entre la fuente generadora de los impulsos eléctricos y el animal, se tom6 en consideración que los componentes con que está construido sean de fácil adquisición yde uso frecuente enel medio. El transductor está integrado por dos partes fundamentales: el cuerpo y el mango descritos en los siguientes numerales [REDVET2005].

\subsubsection{CuerpodelTransductor}

En el diseño se consideró la estructura anat6mica del recto del ovino y caprino, determinando las especificaciones proporcionales en cuanto a longitud, diámetro y disposición de los electrodos del transductor, para ello se consultó la literatura a fin, en donde se menciona cual es la mejor respuesta en cuanto a la disposición yn6mero de los electrodos, encontrando que la mejor forma es la mostrada en la figura 3.

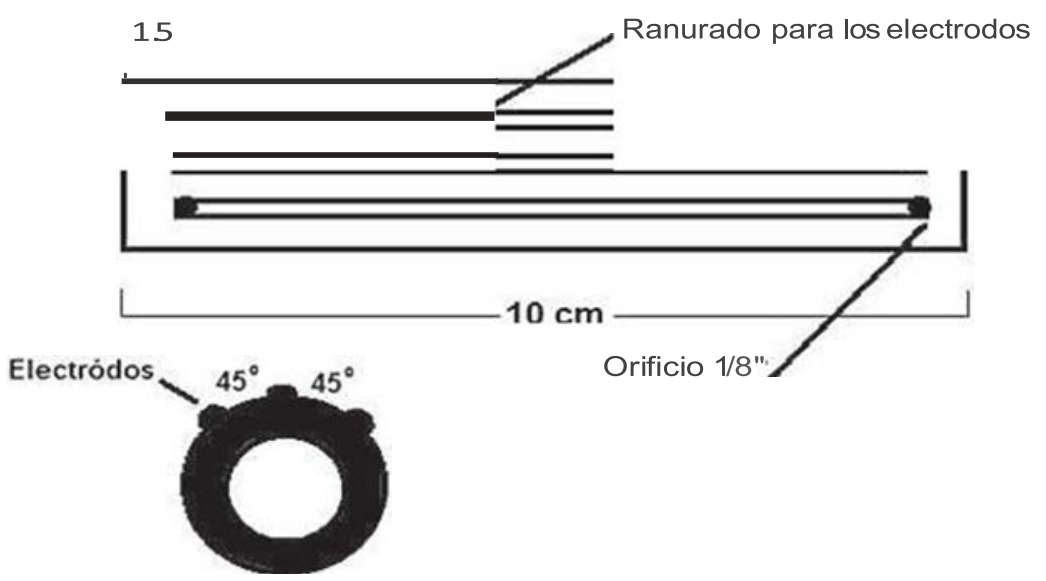

Figura 3. Disposición de Electrodos yTamaño de Sonda Ovinos y Caprinos

\subsection{Mango del Transductor}

El mango está elaborado con piezas de cobre tales como: acoples, tee, niple, codos reducidos, un tapón para uno de sus extremos y para el otro un acople macho de micrófono de 3patas para la conexión de la sonda desde la unidad. Generadora de impulsos. Todas estas partes están unidas con pegamento para obtener la forma adecuada.

\subsection{Sonda Conectora}

La sonda conectora entre la unidad generadora de impulsos y el transductor (sonda rectal), tiene una longitud apropiada de $3 \mathrm{my}$ conectores adecuados para el acople entre las dos partes, cuenta también con una baja impedancia ideal para las transmisión de señales.

Estas onda se compone de tres partes, la primera es el conector Jack macho el cual se conecta directamentea la caja generadora de impulsos, la segunda el conector Canon hembra de tres pines el cual sea copla con la entrada del transductor yla tercera es el cable para micrófono PROTEL encargado de unir estosdos tipos de conectores en el cual se cortocircuitan dos de sus hilos para obtener el acople requerido. 


\section{Resultados}

El Electroeyaculador está construido sobre un gabinete de material metálico, el equipo cuenta con control digital de voltaje desarrollado con un microprocesador que le brinda la posibilidad de ajustar el nivel de tensión en forma precisa, en modo preestablecido y modo ajustable dependiendo de la especie; la programación del microprocesador estimó también los valores límites de voltaje y frecuenciamáximos soportados porcada animal (Tabla 1).

El equipo cuenta con una batería interna que le proporciona suficiente autonomía como para satisfacer la mayoría de sus requerimientos energéticos y con una pantalla LCD para visualizar la especie con la que se desea trabajar, el voltaje a aplicar, el voltaje de la batería, los parámetros detrabajo como tiempo activo, inactivo y frecuencia de operación.

También posee con una serie de botones para el control de trabajo que son: pause, stop, ok, y up o down para el control manual del voltaje. Además de estos botones el equipo tiene un switch para el encendido y apaga.do junto con dos indicadores lumínicos, para el encendido y para la pausa como se ilustra en lafigura4.

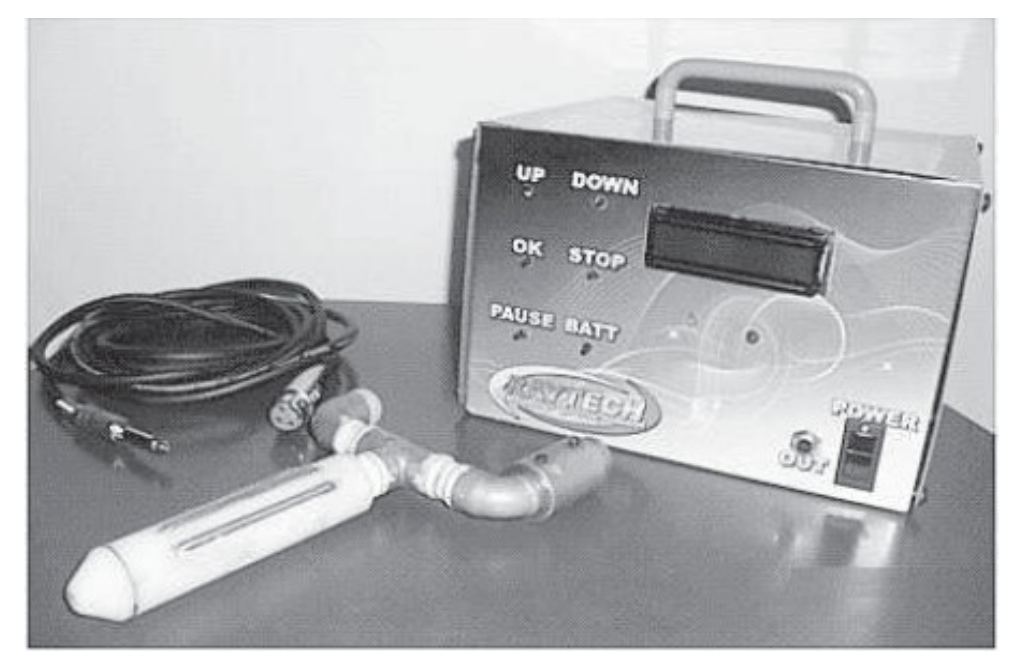

Figura4.RAYTECH Multispecies Electroejaculator

Una vez diseñado y ensamblado el prototipo se realizaron pruebas para hacer ajustes de calibración mediante programación; superada esta etapa sellevó acabo laprueba final empleandoun osciloscopio con aquel instrumento se obtuvieron imágenes de las ondas para cada especie eligiendo valores de voltaje y frecuencia dentro de los rangos establecidos para el prototipo.

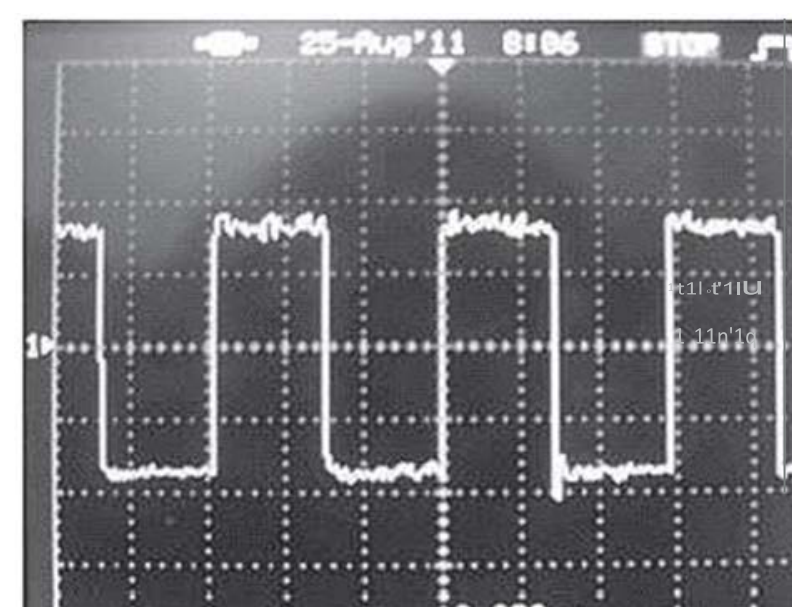

Figura5.Onda Generada por RAYTECHMultispecies Electroejaculator en Modo Ovino/Caprino 
Tabla 2. Informacióndela ondapresentadaenla Figura5.

\begin{tabular}{|c|c|c|c|}
\hline $\mathrm{F}(\mathrm{Hz})$ & 68.57 & $\mathrm{Vmax}(\mathrm{V})$ & 3.68 \\
\hline $\mathrm{T}(\mathrm{ms})$ & 14.58 & $\mathrm{Vmin}(\mathrm{V})$ & -4.24 \\
\hline DutyCycle & $49.38 \%$ & $\operatorname{Vamp}(\mathrm{V})$ & 6.72 \\
\hline $\mathrm{Vpp}(\mathrm{V})$ & 7.84 & $\mathrm{Vrms}(\mathrm{V})$ & 3.36 \\
\hline
\end{tabular}

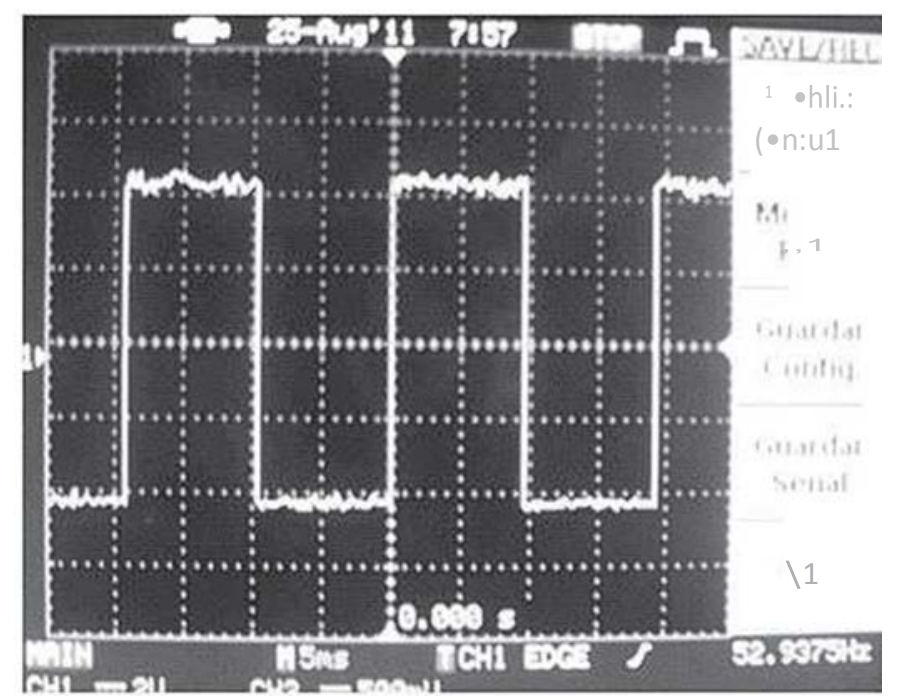

F1gura6. Onda GeneradaporRAYTECH Multispecies Electroejaculator en ModoBobino

Tabla 3. Información dela onda presentada en la Figura 6.

\begin{tabular}{|c|c|c|c|}
\hline $\mathrm{F}(\mathrm{Hz})$ & 52.09 & $\operatorname{Vmax}(\mathrm{V})$ & 5.04 \\
\hline $\mathrm{T}(\mathrm{ms})$ & 19.19 & $\operatorname{Vmin}(\mathrm{V})$ & -4 \\
\hline DutyCycle & $50 \%$ & $\operatorname{Vamp}(\mathrm{V})$ & 8.48 \\
\hline Vpp (V) & 9.04 & $\operatorname{Vrms}(\mathrm{V})$ & 4.29 \\
\hline
\end{tabular}

Las señales mostradas en las figuras 5 y 6 fueron evaluadas y comparadas con estudios preliminares al desarrollo de este proyecto, resultando adecuadas en amplitud y en frecuencia asegurando el bienestar de los animales en los que fue probado el dispositivo, con esto se concluye la aplicación de la técnica de electroeyaculación en las especies ovina, caprina y bobina.

\section{Conclusiones}

Se dotó al dispositivo con una batería recargable de 7Aamperios con el fin de poder usado por largos periodos de tiempo sin necesidad de recargar y de esta manera alargar su vida útil.

Se implementó un sistema de alimentación mediante convertidores DC-DC para obtener la potencia necesaria y una forma de onda particular con el objetivo de lograr la estimulación dentro de los parámetros establecidos por la técnica de electroeyaculación, teniendo así un sistema estable y seguro para el animal.

El desarrollo del prototipo diseñado permitió establecer que teniendo una idea innovadora y utilizando bajo presupuesto se implementó un dispositivo asequible a la comunidad interesada, respondiendo adecuadamente a los requerimientos de la región para la obtención de muestras de esperma limpios de machos de diferentes especies de alta calidad genética, útilpara el proceso de la preservación de especies impulsada por medios artificiales, que permiten impulsar el desarrollo de la región a nivel de la industria pecuaria. 


\section{Referencias Bibliográficas}

MN Sundararaman, J. Kalatharan and MJ Edwin, 2007. Attempts to Achieve Semen Collections from lncapacitated Boer Bucks by Electro-ejaculation .Asian Joumal of Animal and Veterinary Advances, 2: 244- 246.

Revista Electrónica de Veterinaria REDVET. Vol VI No 8. Agosto 2005 Diseño y Construcción de Electroeyaculador Para Ovinos y Caprinos. Universidad Autónoma de Chiapas. Facultad de Medicina Veterinaria Y Zootecnia. México. ISSN 1695-7504
Método
de
la
Electroeyaculación.

http://www latinpedia.net/Ciencia/animalffecnicas-de-extraccion-de-semen-enanimales-domesticos-ad462.htm

Escobar J., 201O. Electroeyaculación Según Jorge Escobar. Consultado el O1defebrero de 2011.

http://jorgeescobar.blog.com/2010/02/22/electroeyaculacion-según/

Diego R. Barrios A., 2009 Evaluación de la Calidad y Capacidad Fecundante de Espermatozoides de la Cola del Epidídimo de Toros Post-Mortem. Post-grado de Reproducción Animal Facultad de Ciencias Veterinarias Universidad Central de Venezuela

Lourdes Tibisay Vilanova F., MY, Dra.; Pedro Pablo Ballarales B., MV, Dr., 2010 La evaluación andrológica: justificación y métodos. Universidad Centroccidental "Lisandro Alvarado", Decanato de Ciencias Veterinarias, Barquisimeto, Estado Lara

M.V., MSc., PhD. Diego R. Barrios A. 2005. Attempts. Consideraciones Básicas Acerca dela Extracción de Semen de Toros Mediante Electroeyaculador. Venezuela Bovina

Paola Díaz, Viviana Fonseca, Patricia Martínez, Andrea Rey, 2003. Inseminación Artificial en Bovinos. Biblioteca Virtual Universal. 